\title{
Collision and impact simulations including porosity
}

\author{
Willy Benz ${ }^{1}$ and Martin Jutzi ${ }^{1}$ \\ ${ }^{1}$ Physikalisches Institut, University of Bern, Siedlerstrasse 5, 3012 Bern, Switzerland \\ email: willy.benz@space.unibe.ch
}

\begin{abstract}
The Smooth Particle Hydrodynamics (SPH) impact code (Benz \& Asphaug 1994) has been developed for the simulation of impacts and collisions involving brittle solids in the strength- and gravity-dominated regime. In the latter regime, the gravitational overburden is used to increase the fracture threshold. In this paper, we extend our numerical approach to include the effect of porosity at a sub-resolution scale by adapting the so-called $P-\alpha$ model (Herrman 1969). Using our extended 3D SPH impact code, we investigated collisions between porous bodies to examine the sensitivity of collisional outcomes to the degree of porosity. Two applications that illustrate the capabilities of our approach are shown: 1) the modeling of a Deep Impact-like impact and 2) the computation of the amount of momentum transferred to an asteroid following the impact of a high velocity projectile.
\end{abstract}

Keywords. Hydrodynamics; shock wave; equation of state

\section{Introduction}

The evidence of the ubiquity of porosity in the structure of small bodies in the present day solar system is rapidly mounting. Spacecraft missions and ground based observations are providing increasing evidence that many or even most asteroids must be, to some degree or another, porous bodies (e.g. Housen \& Holsapple 2003; Britt et al. 2006). Other small bodies in the solar systems like comets are also thought to have highly porous structures. In parallel, the dissipative properties of porous media are invoked more and more as playing an important role in the formation of early planetesimals (e.g. Wurm et al. 2006). Hence, porosity emerges slowly as playing a major role from the time of the formation of the planets to the collisional evolution of the present day solar system.

Despite the growing focus on porosity, our ability to model its effect on the outcome of impacts and collisions remains limited. Wuennemann et al. (2006) proposed recently a strain based model suitable for the use in hydrocodes. In this paper we propose an alternative approach based on the so-called $P$ - $\alpha$ model (Herrmann 1969) and show how to incorporate it into our 3D Smooth Particle Hydrodynamics ( $\mathrm{SPH}$ ) code. We begin by recalling the equations we use in our modeling of brittle solids (section 2) before deriving the new equations and relevant modifications in order to extend our material description towards porous media (section 3). Finally two applications are presented pertaining to impact cratering and momentum transfer in collisions (section 4).

\section{Equations}

Our numerical tool is based on the on the Smooth Particle Hydrodynamic (SPH) method. Since the basic method has already been described many times (see for examples reviews by Benz 1990; Monaghan 1992) we refer the interested reader to these earlier papers. 
The standard gas dynamics SPH approach was extended (see for example Libersky \& Petschek, 1990) to include an elastic-perfectly plastic material description and a fracture model in order to model brittle solids (Benz \& Asphaug, 1994). As our porosity model interfaces with this material description, we begin with a short review of our approach.

\subsection{Elastic perfectly plastic strength model}

The equations to be solved are the well-known conservation equations of elasto-dynamics; they can be found in most standard textbooks. The mass conservation can be written as (using the implicit repeated index summation rule):

$$
\frac{d \rho^{\kappa}}{d t}+\rho \frac{\partial v^{\kappa \lambda}}{\partial x^{\lambda}}=0
$$

where $d / d t$ is the Lagrangian time derivative, $\rho$ the mass density, $v$ the velocity and $x$ the position. The conservation of momentum has the following form:

$$
\frac{d v^{\kappa}}{d t}=\frac{1}{\rho} \frac{\partial \sigma^{\kappa \lambda}}{\partial x^{\lambda}}
$$

where $\sigma^{\kappa \lambda}$ is the stress tensor given by

$$
\sigma^{\kappa \lambda}=S^{\kappa \lambda}-P \delta^{\kappa \lambda}
$$

and $P$ is the pressure, $\delta^{\kappa \lambda}$ the Kroneker symbol and $S^{\kappa \lambda}$ the (traceless) deviatoric stress tensor. Finally, the conservation of energy is given by the equation

$$
\frac{d E}{d t}=-\frac{P}{\rho} \frac{\partial}{\partial x^{\kappa}} v^{\kappa}+\frac{1}{\rho} S^{\kappa \lambda} \dot{\epsilon}^{\kappa \lambda}
$$

where $\dot{\epsilon}$ is the strain rate tensor given by

$$
\dot{\epsilon}^{\kappa \lambda}=\frac{1}{2}\left(\frac{\partial v^{\kappa}}{\partial x^{\lambda}}+\frac{\partial v^{\lambda}}{\partial x^{\kappa}}\right) .
$$

In order to specify the time evolution of the deviatoric stress tensor $S^{\kappa \lambda}$ we adopt Hooke's law and define the time evolution of the deviatoric stress tensor as:

$$
\frac{d S^{\kappa \lambda}}{d t}=2 \mu\left(\dot{\epsilon}^{\kappa \lambda}-\frac{1}{3} \delta^{\kappa \lambda} \dot{\epsilon}^{\nu \nu}\right)+S^{\kappa \lambda} \Omega^{\lambda \nu}+S^{\lambda \nu} \Omega^{\kappa \nu}
$$

with $\mu$ is the shear modulus, and $\Omega$ is the rotation rate tensor:

$$
\Omega^{\kappa \lambda}=\frac{1}{2}\left(\frac{\partial v^{\kappa}}{\partial x^{\lambda}}-\frac{\partial v^{\lambda}}{\partial x^{\kappa}}\right) .
$$

Finally, plasticity is treated using the von Mises yielding criterion.

In order to close the system of equations, an equation of state (EOS) has to be specified which relates density, specific energy and pressure:

$$
P=P(\rho, E)
$$

For the simulations presented in this paper we use the so-called Tillotson equation of state (e.g. Melosh 1989).

\subsection{Fracture}

Brittle materials cannot be modeled using elasticity and plasticity alone because these materials break under tension or shear stress. To take this behavior into account, we use the fracture model based on explicit incipient flaws (Benz \& Asphaug 1995). In this 
model it is assumed that the number density of active flaws at strain $\epsilon$ is given by a Weibull distribution

$$
n(\epsilon)=k \epsilon^{m}
$$

where $k$ and $m$ are the material dependent Weibull parameters. When the local tensile strain has reached the flaw's activation threshold, a crack is allowed to grow at a constant velocity $c_{g}$ which is some fraction of the local sound speed. The half length of a growing crack is therefore

$$
a=c_{g}\left(t-t^{\prime}\right)
$$

where $t^{\prime}$ is the crack activation time. Crack growth leads to a release of local stresses. To model this behavior, we follow Benz \& Asphaug (1995) and introduce a state variable $D$ (damage) which expresses the reduction in strength under tensile loading:

$$
\sigma_{D}=\sigma(1-D)
$$

where $\sigma$ is the elastic stress in the absence of damage and $\sigma_{D}$ is the damage-relieved stress. The state variable $D$ is defined locally as the fractional volume that is relieved of stress by local growing cracks

$$
D=\frac{\frac{4}{3} \pi a^{3}}{V}
$$

where $V=4 / 3 \pi R_{s}^{3}$ is the volume in which a crack of half length $R_{s}$ is growing. Using equation (2.10) and (2.12) we get the following differential equation for the damage growth that we integrate together with the other variables

$$
\frac{d D^{1 / 3}}{d t}=\frac{c_{g}}{R_{s}}
$$

\section{Extension of our numerical method: Including a porosity model}

While porosity at large scales can be modeled explicitly by introducing macroscopic voids, porosity on a scale much smaller than the numerical resolution has to be modeled through a different approach. Our porosity model is based on the so called $P$ - $\alpha$ model initially proposed by Herrmann (1969) and later modified by Carroll \& Holt (1972). The model provides a description of microscopic porosity with pore-sizes beneath the spatial resolution, which is homogeneous and isotropic on the scales we resolve.

\section{1. $P-\alpha$ model}

The basic idea of the $P-\alpha$ model is to separate the volume change in porous material into two parts: the pore collapse on the one hand and compression of the matrix material on the other hand. This separation can be done by introducing the so called distention parameter $\alpha$ which is defined as

$$
\alpha \equiv \frac{\rho_{s}}{\rho}
$$

where $\rho$ is the density of the porous material and $\rho_{s}$ the density of the corresponding solid (matrix) material. Distention is related to porosity as $1-1 / \alpha$. Using the distention parameter $\alpha$, the equation of state can be written in the general form:

$$
P=P(\rho, E, \alpha)
$$

According to Carroll \& Holt (1972), the EOS of a porous material is explicitly given by:

$$
P=\frac{1}{\alpha} P_{s}\left(\rho_{s}, E_{s}\right)=\frac{1}{\alpha} P_{s}(\alpha \rho, E)
$$


where $P_{s}\left(\rho_{s}, E_{s}\right)$ represents the EOS of the solid phase of the material (the matrix). A central assumption in this model is that the pressure depends on density of the matrix material. The specific internal energy $E$ is assumed to be the same in the porous and the solid material $\left(E=E_{s}\right)$. In the $P-\alpha$ - model, the distention is solely a function of the pressure $P$ :

$$
\alpha=\alpha(P)
$$

where $P=P(\rho, E, \alpha)$. The relation between distention and pressure is often assumed to have the following quadratic form:

$$
\alpha=1+\left(\alpha_{0}-1\right) \frac{\left(P_{s}-P\right)^{2}}{\left(P_{s}-P_{e}\right)^{2}} .
$$

where $P_{e}$ and $P_{s}$ are constants. This is obviously a very simple model, however, it is appropriate enough for many applications. A more realistic relation can be obtained experimentally by a one dimensional static compression of the porous material during which the actual distention $\alpha_{m}$ is measured as a function of the applied pressure $P_{m}$. The resulting so-called crush-curve $\alpha_{m}\left(P_{m}\right)$ then provides the required relation between distention and pressure and can be used directly in the code.

In a quasi-static compression, the energy contribution to the pressure is very small and in the porous regime (where $\alpha>1$ ), even mostly negligible. Therefore, the pressure can be approximated by $P=P(\rho, \alpha)$ and consequently, we can transform the function $\alpha(P[\rho, \alpha])$ into $\alpha=\alpha(\rho)$. This transformation can be done in both cases where either analytical relations $(\alpha(P))$ or crush-curves measured experimentally $\alpha_{m}\left(P_{m}\right)$ have been used. As we will see below, the use of $\alpha(\rho)$ instead of $\alpha(P)$ has some significant advantages. Therefore, in practice we are actually using $\alpha=\alpha(\rho)$ Using $\alpha(\rho)$, the time evolution of the distention parameter is simply given by

$$
\dot{\alpha}=\frac{d \alpha}{d \rho} \dot{\rho}
$$

which is pressure independent. The corresponding relation for the case where the energy contribution cannot be neglected, $\alpha=\alpha(P)$, is given by

$$
\dot{\alpha}(t)=\frac{\dot{E}\left(\frac{\partial P_{s}}{\partial E_{s}}\right)+\alpha \dot{\rho}\left(\frac{\partial P_{s}}{\partial \rho_{s}}\right)}{\alpha+\frac{d \alpha}{d P}\left[P-\rho\left(\frac{\partial P_{s}}{\partial \rho_{s}}\right)\right]} \cdot \frac{d \alpha}{d P}
$$

which depends upon the pressure. This pressure-dependence of $\dot{\alpha}$ can cause numerical instability because the pressure itself depends on $\alpha$ (equation 3.3). For this reason, other alternative models such as the strain based porosity model (Wuennemann et al. 2006) have been explored. However, we did not find these instabilities when using $\alpha=\alpha(P)$. The main reason that we use $\alpha=\alpha(\rho)$ instead of $\alpha=\alpha(P)$ is its applicability to relate the distention $\alpha$ to strength.

\subsection{Porosity and strength}

As we have discussed in the previous section, the pressure is calculated using the matrix density $\rho_{s}$ instead of $\rho$. Therefore, the deviatoric stress tensor has to be modified as well. In order to compute the time evolution of $S^{\kappa \lambda}$ as a function of the matrix variables, we introduce the following correction factor:

$$
f \equiv \frac{\dot{\rho}_{s}}{\alpha \dot{\rho}}
$$


In fact, this factor relates the velocity divergence of the matrix to that of the porous material:

$$
[\vec{\nabla} \vec{v}]_{s}=f \vec{\nabla} \vec{v}
$$

where $[\vec{\nabla} \vec{v}]_{s}$ is defined to satisfy the continuity equation of the matrix:

$$
\dot{\rho}_{s}=-\rho_{s}[\vec{\nabla} \vec{v}]_{s}
$$

Using $\alpha=\alpha(\rho)$ and $\dot{\rho}_{s}=\alpha \dot{\rho}+\dot{\alpha} \rho, f$ has the simple form:

$$
f=\frac{\rho}{\alpha} \frac{d \alpha}{d \rho} .
$$

Using $\alpha=\alpha(P)$, we obtain $f=f(P, \rho, \dot{\rho}, E, \dot{E})$ which (in the case of a small $\dot{\rho}$ and a large $\dot{E}$ ) can lead to numerical instabilities. This is the main reason that we are actually using $\alpha=\alpha(\rho)$. The factor $f$ is used to compute the corrected time evolution of $S^{\kappa \lambda}$ :

$$
\frac{d S^{\kappa \lambda}}{d t} \rightarrow f \frac{d S^{\kappa \lambda}}{d t}
$$

In addition, the deviatoric stress tensor $S^{\kappa \lambda}$ is multiplied by $\alpha^{-1}$ as it is done with the pressure $P$ for the same reason (see Carroll \& Holt 1972. We finally write the time evolution of $S^{\kappa \lambda}$ in the following form:

$$
\frac{d}{d t}\left[\frac{1}{\alpha} S^{\kappa \lambda}\right]=\frac{1}{\alpha} \frac{d S^{\kappa \lambda}}{d t}-\frac{1}{\alpha^{2}} S^{\kappa \lambda} \frac{d \alpha}{d t}
$$

where $d S^{\kappa \lambda} / d t$ is modified according to (3.12).

\subsection{Porosity and damage}

Porosity does not only affect the stress behavior it also has to be taken into account to compute the state variable damage.

Compression of a porous material beyond the elastic limit is accompanied by breaking cell walls. We model this crushing behavior by relating the distention to the state variable damage $(D)$. Since both, damage $D$ and distention $\alpha$, are defined as a volume ratio (equations 2.12 and 3.1, respectively) we assume for simplicity (other forms will be investigated in the future) a linear relation between $D$ and $\alpha$. The conditions: $D=0$ at $\alpha=\alpha_{0}$, and $D=1$ when all pores have been crushed $(\alpha=1)$, lead to the following expression:

$$
D=1-\frac{(\alpha-1)}{\left(\alpha_{0}-1\right)} .
$$

The time evolution of $D^{1 / 3}(\alpha)$ is given by

$$
\frac{d D^{1 / 3}}{d t}=\frac{d D^{1 / 3}}{d \alpha} \frac{d \alpha}{d t}
$$

and using equation (3.14) we obtain

$$
\frac{d D^{1 / 3}}{d t}=\frac{1}{3}-\left[\frac{\alpha-1}{\alpha_{0}-1}+1\right]^{-\frac{2}{3}}-\frac{1}{\alpha_{0}-1} \frac{d \alpha}{d t} .
$$

We now have two equations describing damage growth: the first treats damage under tension (2.13) while the second (3.16) is related to the compression of the (porous) material. In order to get the total grow of damage, we build the sum of these two differential 
equations:

$$
\left[\frac{d D^{1 / 3}}{d t}\right]_{\text {total }}=\left[\frac{d D^{1 / 3}}{d t}\right]_{\text {tension }}+\left[\frac{d D^{1 / 3}}{d t}\right]_{\text {compression }}
$$

\subsection{Material parameters}

All parameters used by our porosity model are material parameters which can in principle be measured; some of them quite easily (e.g. the crush-curve) even though this is rarely done in practice. Others, such as Weibull parameters, shear strength etc. are more difficult to measure.

The lack of an experimentally determined reliable database of relevant material parameters is actually one of the most limiting factor in our model. In particular, the thorough testing of the model by comparison with experiments is rendered particularly difficult if all material properties have not been measured properly. Freely choosing the missing values so as to match the experiment is not a satisfactory approach for an ab initio method such as ours. Unfortunately, this is often the only alternative we have.

\section{Applications of our model}

Although our method still needs to be rigorously tested, the first calculations show very encouraging results. For instance, we simulated the laboratory impacts in porous material carried out by Housen \& Holsapple (2003). We were able to reproduce the shape of the crater produced in these experiments (Jutzi 2004).

As an illustration of the capabilities of our code, we show in this paper the results of two sets of simulations. In the first one, we computed the impact of a Deep Impactlike projectile onto a icy target of different porosity. In this calculation, we did not try to obtain an exact match by fiddling with all the parameters but rather used standard inputs to see how porosity was affecting the results. The second example illustrates our efforts to compute the amount of momentum transferred to an asteroid by the impact of a high speed projectile as a function of the target porosity.

\subsection{Modeling a Deep Impact-like experiment}

We carried out simulations of a Deep Impact-like impact on comet Temple-1 using three different initial porosities $(0 \%, 33 \%$ and $67 \%)$ for the target comet. Only a small part of Temple-1 was modeled (half sphere with a radius of $22 \mathrm{~m}$ ). As target material we used pre-damaged (strenghtless) ice with a corresponding initial distention of 1.0, 1.5 and 3.0. The impactor was modeled as a $370 \mathrm{~kg}$ aluminum sphere impacting at an angle of 30 degree (from horizontal) with a velocity of $10 \mathrm{~km} / \mathrm{s}$. For all these simulations we used $700^{\prime} 000$ particles for the target and 20 particles for the projectile. This results in a spatial resolution of $36 \mathrm{~cm}$ (target) and $22 \mathrm{~cm}$ (projectile), and particles masses of 9.5 to $29 \mathrm{~kg}$ (target) and $19 \mathrm{~kg}$ (projectile).

Figure 1 shows the outcome of the simulation after $50 \mathrm{~ms}$. In these 2-D slices of the 3 -D target, the color shows the vertical velocity of the particles $(\mathrm{cm} / \mathrm{s})$. Note that the escape velocity of Temple 1 is about $200 \mathrm{~cm} / \mathrm{s}$. Obviously, there is much more material ejected in the non-porous than in the porous cases. The difference of ejected material in the simulations with porosity is only small. However, since for $\alpha_{0}=1.5$, the particle mass is twice the mass of the particles with $\alpha_{0}=2.0$, slightly more mass is ejected in the simulation with the lower porosity. For illustration, we also show the actual value of the distention $\alpha$ in the case $\alpha_{0}=3.0$ (figure 2). 

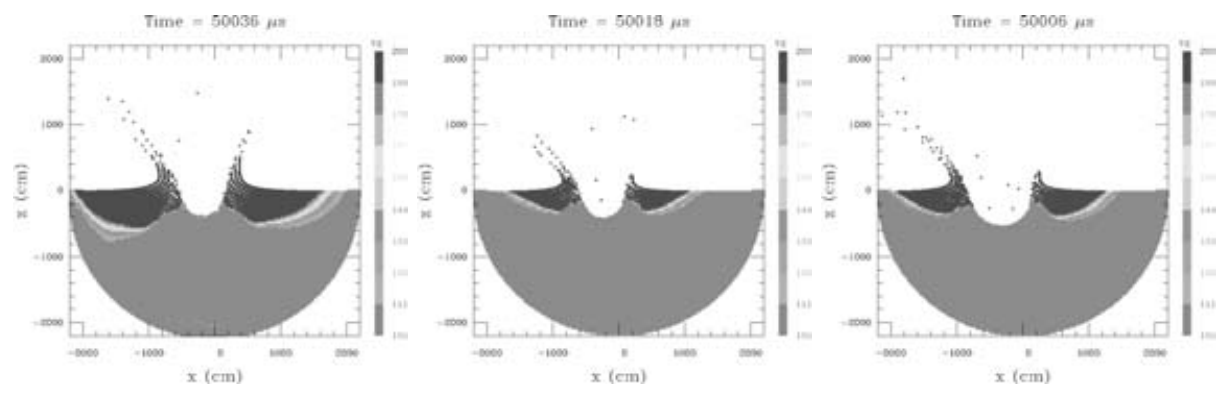

Figure 1. Simulation of a Deep Impact-like impact using different initial distention: $\alpha_{0}=1.0$ (left), $\alpha_{0}=1.5$ (middle), $\alpha_{0}=3.0$ (right). The colors label the z-component of the velocity, red indicates a velocity higher than the escape velocity.



Figure 2. A measure of the compaction is provided by the actual value of the distention $\alpha$. The initial distention of $\alpha_{0}=3$ is decreased to $\alpha=1$ in a small zone around the crater. With increasing distance from the crater, distention increases until $\alpha=\alpha_{0}$

A quantity of interest is the amount of mass ejected with a velocity higher than a certain velocity. Figure 3 shows the corresponding relation obtained in our simulations. If we assume the escape velocity to be $v_{e s c}=2 \mathrm{~m} / \mathrm{s}$, we get the total mass ejected: $3.20 \times 10^{6} \mathrm{~kg}, 0.52 \times 10^{6} \mathrm{~kg}$, and $0.49 \times 10^{6} \mathrm{~kg}$ for $\alpha_{0}=1.0,1.5$ and 3.0 respectively. Again there is a big difference between the nonporous and porous simulations and a smaller difference between the two porous simulations. The reason could be that we only changed one parameter in our porosity model $\left(\alpha_{0}\right)$. Further investigations will be done to examine the sensitivity of the simulation outcome on the model parameters (crush-curve).

\subsection{Momentum transfer}

The change in orbit of an asteroid resulting from the impact of a projectile depends upon the momentum transferred during the collision. To compute this amount is not straight forward as it is largely determined by the amount of material ejected from the impact crater. This amount as well as its velocity distribution are functions of the internal structure and material characteristics of the target (at least in the impact area). Hence, 


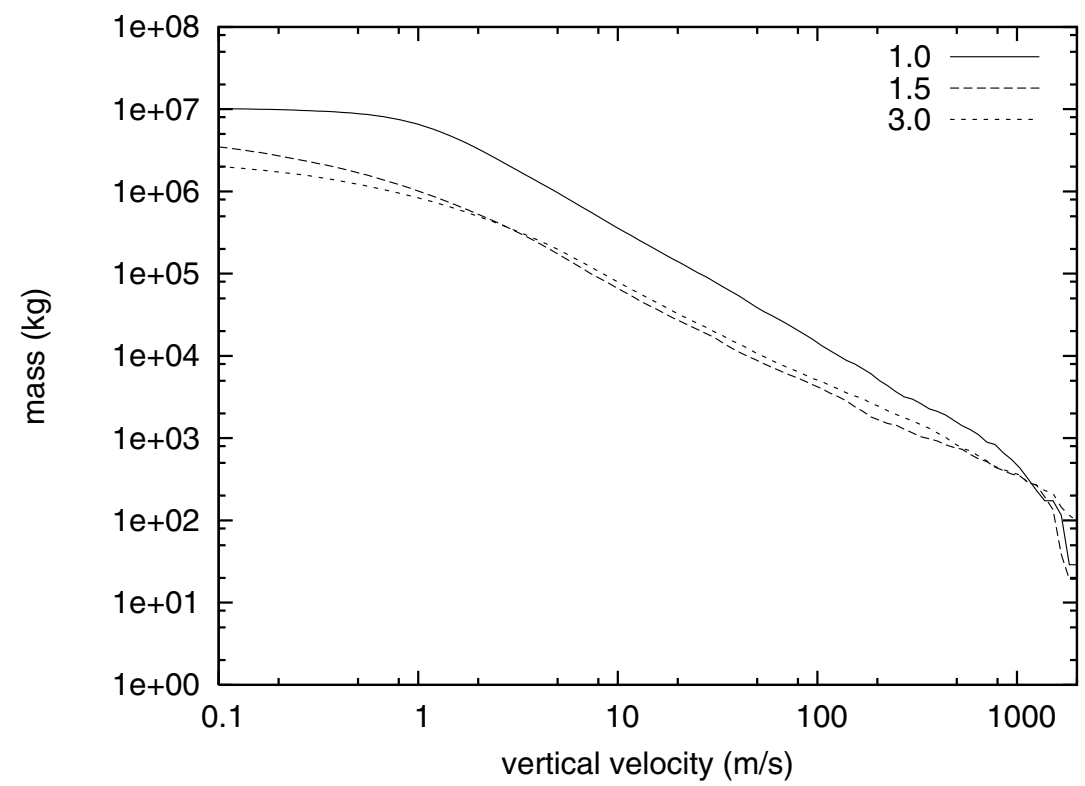

Figure 3. Deep Impact-like simulation: cumulated mass as a function of the ejection velocity for different initial distentions $\alpha_{0}$

the degree of porosity of the asteroid will play a key role in determining the size of impact required to achieve a given orbital change.

To examine how porosity might affect the momentum transfer achieved in a given collision, we simulate impacts of a space-craft with a velocity of $10 \mathrm{~km} / \mathrm{s}$ into an asteroid. The space-craft is modeled as a spherical solid body with a mass of $400 \mathrm{~kg}$ and a density of $5.5 \mathrm{~g} / \mathrm{cm}^{3}$. Since the expected crater is small compared to the overall size of the asteroid, we only model a small fraction of the target (half sphere of $40 \mathrm{~m}$ radius). The material type is taken to be basalt and targets with $0 \%, 9.1 \%, 33 \%$ and $67 \%$ porosity (which corresponds to a initial distention of $1.0,1.1,1.5,3.0)$ are investigated. We use $1^{\prime} 400^{\prime} 000$ particles for the target and 15 for the projectile, resulting in a spatial resolution of 51 $\mathrm{cm}$ (target) and $19 \mathrm{~cm}$ (projectile) and a particle mass of $85-256 \mathrm{~kg}$ (target) and $26 \mathrm{~kg}$ (projectile). The high mass of target particles is a result of the large target size which is required to make sure that the reflection of the shock-wave at the border of the half sphere does not affect the results.

To compute the momentum transferred to the target, we determined the mass and velocity of the material reaching velocities equal or in excess of the escape velocity for each simulation. Using momentum conservation, the transferred momentum is then given by $P_{\text {trans }}=P_{\text {proj }}+P_{\text {ejecta }}$, or in units of of $P_{\text {proj }}: P_{\text {trans }}=1+P_{\text {ejecta }}$.

Figure 4 shows $P_{\text {trans }}$ obtained in our simulations as a function of time. There are two main differences between the non-porous and porous simulations. First, there is a peak at early times in the non-porous case which does not occur in porous targets. This peak is caused by the acceleration of particles behind the shock-front due to the rarefaction wave. After a certain time (indicated by the curve's peak) the velocity caused by the rarefaction wave drops below the ejection limit (escape speed), hence the rapid decrease. Using porous material, the shock wave is strongly damped and it also travels much slower. Therefore, the described effect does not occur. 

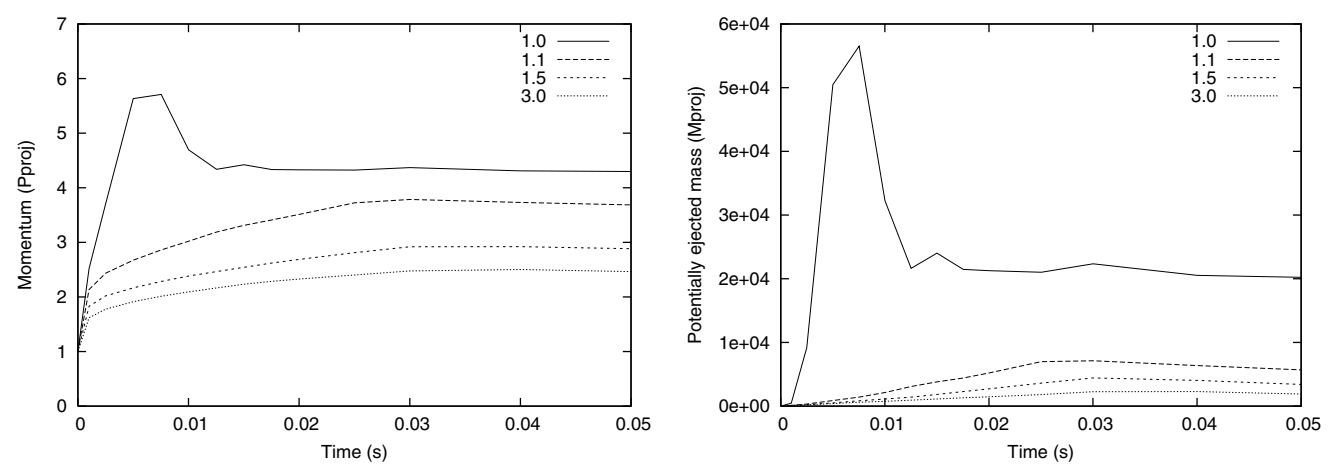

Figure 4. Left: The amount of momentum transferred in an impact to the target decreases with increasing porosity (i.e., increasing distention). Right: The small amount of momentum transferred to porous targets can be explained by the small amount of ejected mass.

The most important difference between the non-porous and porous simulations is the final amount of transferred momentum which (as expected) decreases with increasing porosity. The lower amount of transferred momentum using porous material can be explained by the smaller amount of mass ejected from targets with increasing porosity. This is shown in figure 4 where the potentially ejected mass $\left(v_{z}>v_{e s c}\right)$ is plotted as a function of time.

In order to show that the different behavior of non-porous and porous material is not merely due to the different initial density, we compare a simulation where $\alpha_{0}=1.5$ with a simulation where we do not model porosity but use the same the initial density: $\rho_{0} \rightarrow \rho_{0} / 1.5$. As it can be seen in figure 5 , the effect porosity is not to simply change the bulk density but porosity really affects the dynamics in the sense that crushing the pores represents an important sink of energy.

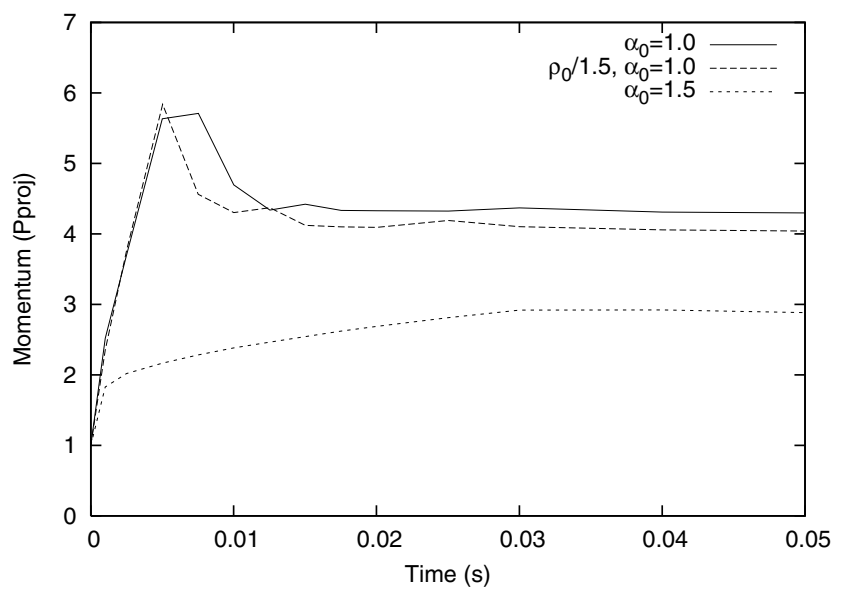

Figure 5. Comparison of a simulation with $\alpha_{0}=1.5$ and a simulation with the same initial density $\rho_{0} \rightarrow \rho_{0} / 1.5$ but without explicit modelling of porosity.

\section{Conclusions}

In this paper, we have presented a new approach to model small scale porosity in brittle solids that can be coupled to a $3 \mathrm{D} \mathrm{SPH}$ hydrocode in order to simulate impacts 
and collisions involving porous bodies. In practice, the implementation of our model does not consume excessive CPU time and is easily implemented in a parallel code (porosity is a local property) so that simulations involving multi-million particles can readily be performed. In fact, extensive testing has shown that high resolution is really needed to obtain converged solutions in the case of simulations involving fracturing and/or porosity.

Testing of the code including porosity is made difficult by the lack of well described experiments in which all relevant material parameters have been measured and published. More often than none, the missing parameters can be chosen freely. The fact that, with a reasonable choice for these, a good agreement can be found lends some confidence to the model but is not completely satisfactory.

Despite these difficulties, the relative effect of porosity on the outcome of an impact or collision can be studied by keeping, for example, all other parameters fixed. By means of two examples, we illustrate how strongly porosity can affect the dynamics leading to important changes in the event's outcome. In the first example, we show how the amount of ejecta following a Deep Impact-like event is decreasing with increasing porosity. Our results are indeed consistent (given the simplicity of the model) with values inferred from the experiment (see A'Hearn this volume). The second example deals with the amount of momentum transferred to a target asteroid by the impact of a high-speed projectile. We showed that this amount is considerably reduced (more than a factor 2) by increasing distension from 1 to 3 . The cause of this reduction is to be found in the corresponding decrease of ejected matter with increasing distension. We also show that taking into account the dissipation provided by porosity is important. Simply reducing the bulk density is not a proper approach to model porous media.

\section{Acknowledgements}

The authors gratefully acknowledge partial support from the Swiss National Science Foundation.

\section{References}

Benz, W. 1992, in: J.R. Büchler (ed.), The numerical modelling of nonlinear stellar pulsations: Problems and prospects (Springer: Berlin), p. 269.

Benz, W. \& Asphaugh, E. 1994, Icarus 107, 98

Benz, W. \& Asphaugh, E. 1995, Comp. Phys. Comm. 87, 253

Britt, D. T., Consolmagno, G. J. \& Merline, W. J. 2006, 37th Annual Lunar and Planetary Science Conference, 2214

Carroll, M. M. \& Holt, A. C. 1972, J. Appl. Phys. 43, 759

Herrmann, W. 1969, J. Appl. Phys. 40, 2490

Housen, K. R. \& Holsapple K. A. 2003, Icarus 163, 102

Jutzi, M. 2004, Diploma thesis, University of Bern

Libersky, L. D. \& Petschek, A. G. 1990, in Lecture Notes in Physics 395, 248

Melosh, H. J. 1989, Impact cratering: A geologic process (Oxford: Oxford University Press)

Monaghan, J. J. 1992, Annu. Rev. Astron. Astrophys. 30, 543

Wuennemann, K., Collins, G. S. \& Melosh, H. J. 2006, Icarus 180, 514

Wurm, G., Paraskov, G. \& Krauss, O. 2005, Icarus 178, 253 